\title{
Catching COVID: Engineering Peptide-Modified Surface-Enhanced Raman Spectroscopy Sensors for SARS-CoV-2
}

\author{
Taylor D. Payne, ${ }^{\S}$ Stephen J. Klawa, ${ }^{\S}$ Tengyue Jian, Sang Hoon Kim, Micah J. Papanikolas, \\ Ronit Freeman,* and Zachary D. Schultz*
}

Cite This: https://doi.org/10.1021/acssensors.1c01344

Read Online

ACCESS |

山ll Metrics \& More

回 Article Recommendations

Supporting Information

ABSTRACT: COVID-19 remains an ongoing issue across the globe, highlighting the need for a rapid, selective, and accurate sensor for SARSCoV-2 and its emerging variants. The chemical specificity and signal amplification of surface-enhanced Raman spectroscopy (SERS) could be advantageous for developing a quantitative assay for SARS-CoV-2 with improved speed and accuracy over current testing methods. Here, we have tackled the challenges associated with SERS detection of viruses. As viruses are large, multicomponent species, they can yield different SERS signals, but also other abundant biomolecules present in the sample can generate undesired signals. To improve selectivity in complex biological environments, we have employed peptides as capture probes for viral proteins and developed an angiotensin-converting enzyme 2 (ACE2) mimetic peptide-based SERS sensor for SARS-CoV-2. The unique

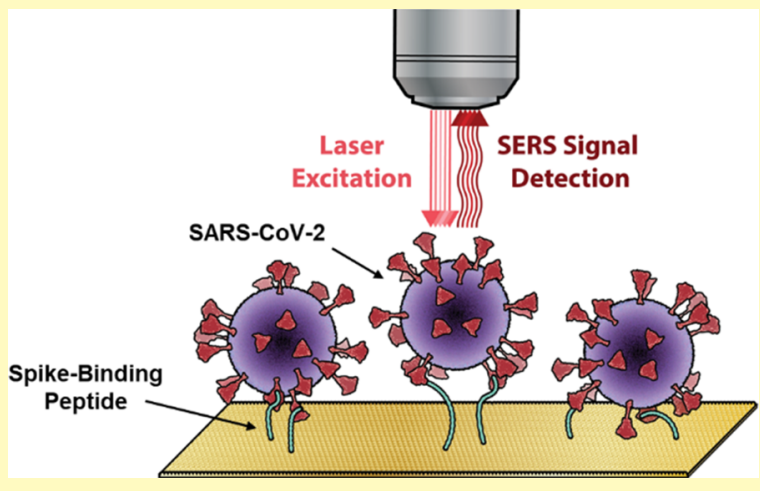
vibrational signature of the spike protein bound to the peptide-modified surface is identified and used to construct a multivariate calibration model for quantification. The sensor demonstrates a $300 \mathrm{nM}$ limit of detection and high selectivity in the presence of excess bovine serum albumin. This work provides the basis for designing a SERS-based assay for the detection of SARS-CoV-2 as well as engineering SERS biosensors for other viruses in the future.

KEYWORDS: SERS, biosensor, COVID-19, SARS-CoV-2, peptide, virus, biomimetic

$\mathrm{T}$ he swift and accurate diagnosis of COVID-19 remains a critical factor in preventing the spread of the disease, which to date has surpassed 176 million cases worldwide. ${ }^{1}$ Current detection methods include polymerase chain reaction (PCR), which requires long run times and significant sample preparation, and antibody testing, which suffers from high false negative rates and detects an immune response from the virus rather than the virus itself. ${ }^{2}$ In addition, diagnostic assays that are rapidly adaptable to virus mutations and variants are needed, as the rapid proliferation of the virus can alter the nucleic acid sequence or surface marker of the virus, rendering it undetectable by current methods. There remains a need for a quick, selective, and error-free sensor for SARS-CoV-2.

Surface enhanced Raman spectroscopy (SERS) is a rapid, sensitive vibrational spectroscopy technique that requires minimal sample preparation and gives a highly specific molecular fingerprint. ${ }^{3}$ Recognized in 1977, ${ }^{4}$ SERS takes advantage of the properties of noble metal nanostructures, which produce a localized electric field upon laser excitation, giving enhanced Raman signals from analytes on the surface. SERS provides a sensitive response, which allows for quantification, and can yield low limits of detection, even down to the single molecule level. ${ }^{5}$ The spectrum contains information about the identities of the adsorbed species and their orientations on the nanostructure surface, providing chemically specific signals to identify analytes. SERS has emerged as a popular analytical method for sensing biomolecules, including proteins and viruses. ${ }^{6}$ This technique could be used to develop a quantitative assay for SARS-CoV-2 that would provide immediate and accurate COVID test results for patients around the world.

Engineering an effective SERS sensor requires tailoring surface chemistry to enhance the SERS response and ensure a reproducible, quantitative diagnostic. While SERS can detect trace amounts of target molecules, other components present in biological assays can generate interference and complicate detection. ${ }^{7}$ Proper modification of the nanostructured surface with a capture agent can increase affinity for a specific analyte in complex environments. ${ }^{8,9}$ Antibodies are a common recognition element for sensing virus particles with SERS, ${ }^{10}$ but they are large and bulky, producing complex SERS spectra

Received: June 25, 2021

Accepted: August 24, 2021 
with a great deal of signal resulting from the antibody itself and also suffering from the same complications as existing immunosensing strategies. ${ }^{7}$ Instead, smaller motifs such as DNA aptamers or peptides can be utilized as capture probes to target viruses. ${ }^{11-13}$ These small capture agents are advantageous due to their facile synthesis and better stability compared to antibodies. Given the near-field enhancement of SERS signals, small capture molecules attached to the nanostructures can concentrate the analyte at the surface where the electric field enhancement is the greatest and thus improve limits of detection. Additionally, the SERS signal depends on the orientation of the analyte on the substrate. Capture molecules can uniformly orient the analyte on the surface, improving signal reproducibility, increasing selectivity, and enabling quantitative models. ${ }^{14}$ Recently, this approach has successfully detected the influenza virus and HIV-1 DNA by SERS. ${ }^{12,13}$

Generating a reproducible response from a specific analyte enables machine learning algorithms to analyze the observed signal. $^{15-18}$ As opposed to performing univariate analysis on a peak characteristic of the analyte, limits of detection and selectivity can be improved by utilizing multivariate analysis techniques, such as multivariate curve resolution (MCR), to create a calibration model based on the entire spectral signature. ${ }^{19}$ Using a simple capture agent reproducibly orients the desired analyte on the surface, ${ }^{14}$ giving rise to a conserved SERS signal that can be extracted from complex mixtures. With appropriate preprocessing, such as normalizing the SERS intensity to an internal standard, quantitative calibration models can be obtained. ${ }^{20-22}$ Building models based on a target spectrum can significantly enhance selectivity in the presence of similar, potentially interfering molecules. ${ }^{23,24}$

Here, we present a peptide-based SERS sensor for SARSCoV-2 that may provide faster, more accurate detection and aid in stopping the spread of COVID-19. SARS-CoV-2 binds to the ACE2 receptor through its spike surface proteins. ${ }^{25}$ The receptor binding domain of the spike protein and its interaction with ACE2 have been thoroughly characterized through cryo-electron microscopy and molecular dynamics simulations. $^{26,27} \mathrm{We}$ have synthesized a peptide sequence derived from the domain of ACE2 that binds the receptor binding domain $(\mathrm{RBD})^{28}$ to selectively capture the SARSCoV-2 spike protein on a SERS active substrate. We demonstrate that SERS can be used to detect the binding of the spike protein to this peptide. We use the resulting SERS signal from the peptide-modified substrate to identify the vibrational signature of the spike protein. This sensor enhances the selectivity and enables detection of the spike protein in heterogeneous samples. In addition, we were able to quantify the spike protein through the use of multivariate analysis, yielding limits of detection in the nanomolar range. This sensor offers a new approach that may be effective for rapid detection of SARS-CoV-2 and other future pathogens.

\section{EXPERIMENTAL METHODS}

Chemicals and Materials. All purchased chemicals were used without further purification. Rink amide MBHA resin was purchased from Chem-Impex. Fmoc-(PEG) $)_{4}-\mathrm{OH}$ was purchased from PurePEG. Alginate, Fmoc-protected amino acids, biotin, HBTU, PyBOP, Oxyma pure, DIC, TFA, TIPS, BSA, $\mathrm{NaBH}_{4}$, and 2-mercaptoethanol were purchased from Sigma-Aldrich. TCEP $\mathrm{HCl}$, DMSO, and $1 \times \mathrm{PBS}$ were purchased from Thermo Fisher. Baculovirus insect-derived SARS-CoV-2 Spike RBD-His (Cat\#: 40592-V08B), SARS-CoV-2 Spike S1 + S2-His (Cat\#: 40589-V08B1), SARS-CoV Spike RBD-His
(Cat\#: 40150-V08B2), and MERS-CoV Spike RBD-His (Cat\#: 40071-V08B1) were purchased from SinoBiological.

Peptide Synthesis and Purification. All peptides were synthesized using an automated standard fluoren-9-ylmethoxycarbonyl (Fmoc) solid-phase peptide synthesis method (Liberty Blue, CEM) on rink amide MBHA resin (100-200 mesh, $0.77 \mathrm{mmol} / \mathrm{g}$ ). Peptides were cleaved from the resin using a solution of $95 \%$ trifluoroacetic acid (TFA), $2.5 \%$ triisopropylsilane (TIPS), and $2.5 \% \mathrm{dH}_{2} \mathrm{O}$. The acid was evaporated, and the crude peptide was purified using reversephase HPLC (Shimadzu UFLC, Ultra C18 $5 \mu \mathrm{M}, 100 \times 10 \mathrm{~mm}$ column) with a gradient of $0.1 \%$ TFA in water (solvent A) and acetonitrile (solvent B) over $50 \mathrm{~min}$. Purified peptides were lyophilized and stored at $-20{ }^{\circ} \mathrm{C}$. Purity was confirmed by electrospray ionization mass spectrometry and HPLC.

X-ray Photoelectron Spectroscopy (XPS). Gold-coated Silmeco SERS substrates were pre-cleaned by heating to $175^{\circ} \mathrm{C}$ for $10 \mathrm{~min}$ under a stream of nitrogen gas. A $1 \mathrm{mM}$ solution of cysteine-modified peptide (20\% DMSO, $2 \mathrm{mM}$ TCEP, in water) was reduced for $1 \mathrm{~h}$. The substrates were submerged in the reduced peptide solution overnight to functionalize the gold surface. Substrates were then washed with $1 \mathrm{~mL}$ of sterile water and backfilled by exposure to 100 $\mu \mathrm{M} 2$-mercaptoethanol for $1 \mathrm{~h}$. Substrates were rinsed in sterile water and dried before use. To assess protein binding, modified substrates were incubated for $1 \mathrm{~h}$ with $1 \mu \mathrm{M}$ RBD in PBS followed by three washes in water.

XPS spectra were recorded using a Kratos Axis Ultra DLD X-ray photoelectron spectrometer with a monochromatic $\mathrm{Al} \mathrm{K} \alpha$ source at $150 \mathrm{~W}$. The spot size area was $300 \mu \mathrm{m} \times 700 \mu \mathrm{m}$. Survey scans over a binding energy range of $0-1200 \mathrm{eV}$ were taken for each sample with a constant detector pass energy range of $80 \mathrm{eV}$ followed by a highresolution XPS measurement (20 eV pass energy) for quantitative determination of binding energy and atomic concentration. Background subtraction, peak integration, and fitting were carried out using Kratos software. To convert peak areas to surface concentration, default instrument sensitivity factors were used $(\mathrm{N}=0.477, \mathrm{C}=$ $0.278, \mathrm{O}=0.780, \mathrm{~S}=0.668, \mathrm{Si}=0.328$, and $\mathrm{Au}=6.250$ ).

Confocal Laser Scanning Microscopy (CLSM). Surfaces were modified with peptide and RBD as described above and were then fixed with $2 \%$ paraformaldehyde and $2.5 \%$ glutaraldehyde in PBS for $20 \mathrm{~min}$. The fixed substrates were blocked with $2 \%$ BSA for $30 \mathrm{~min}$, washed with PBS, and incubated with primary CR3022 antibody (recombinant anti-SARS-CoV-2 spike chimeric rabbit monoclonal antibody, $10 \mu \mathrm{g} / \mathrm{mL}$ ) at room temperature for $1 \mathrm{~h}$. The substrates were washed with PBS and incubated for another hour with goat antirabbit IgG-Alexa488 $(4 \mu \mathrm{g} / \mathrm{mL})$. The immunolabeled substrates were washed with sterile water before being mounted on a glass coverslip for imaging. Images were taken on a Zeiss 710 laser scanning confocal microscope.

Scanning Electron Microscopy (SEM). For SEM, substrates were cleaned or modified with peptide as described above and were then dried under a stream of nitrogen gas. For immunolabeled SEM surfaces, substrates modified with SBP-PEG 4 were incubated for $1 \mathrm{~h}$ with $1 \mu \mathrm{M}$ RBD in PBS and washed three times. The surface was blocked with $2 \%$ BSA for 30 min followed by incubation with primary CR3022 anti-RBD antibody (recombinant anti-SARS-CoV-2 spike chimeric rabbit monoclonal antibody, $10 \mu \mathrm{g} / \mathrm{mL}$ ) for $1 \mathrm{~h}$ at room temperature. Unbound primary antibody was washed away, and the surface was incubated with goat anti-rabbit IgG conjugated to $20 \mathrm{~nm}$ AuNPs for $1 \mathrm{~h}$. The AuNP-labeled substrate was washed three times with sterile water before imaging. Substrates were mounted on SEM stubs using double-sided copper tape. Images were collected using an FEI Helios 600 Nanolab Dual Beam System operating at a $5.00 \mathrm{kV}$ accelerating voltage.

Biolayer Interferometry (BLI). Dissociation constants $\left(K_{\mathrm{d}}\right)$ of peptides were measured by biolayer interferometry (BLI; ForteBio Octet Red 384). Streptavidin BLI tips were functionalized with 2.5 $\mu \mathrm{M}$ biotin-SBP and biotin-SBP-PEG 4 in $1 \times$ kinetic buffer $(1 \times$ PBS with $0.1 \%$ BSA and $0.05 \%$ Tween 20 ). Peptide-modified tips were incubated with various concentrations of SARS-CoV-2 RBD from 0 to $5 \mu \mathrm{M}$ for $400 \mathrm{~s}$. Then, dissociation was measured for $600 \mathrm{~s}$. 


\section{A. (i) No Virus Present}

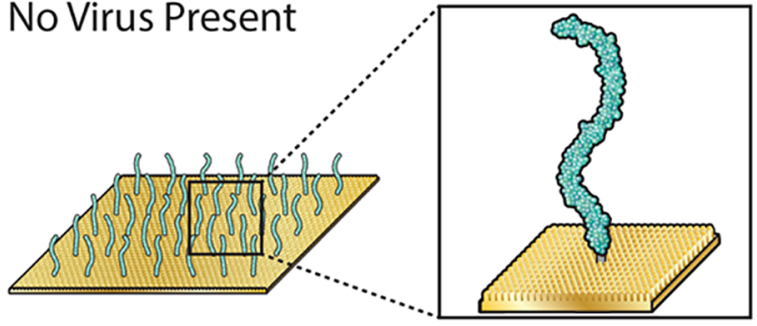

(ii) Virus Present

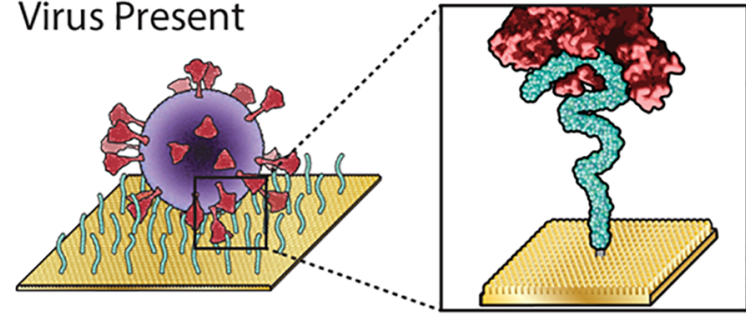

B.

C - $[\text { PEG }]_{n}$ - IEEQAKTFLDKFNHEAEDLFYQS-NH ${ }_{2}$

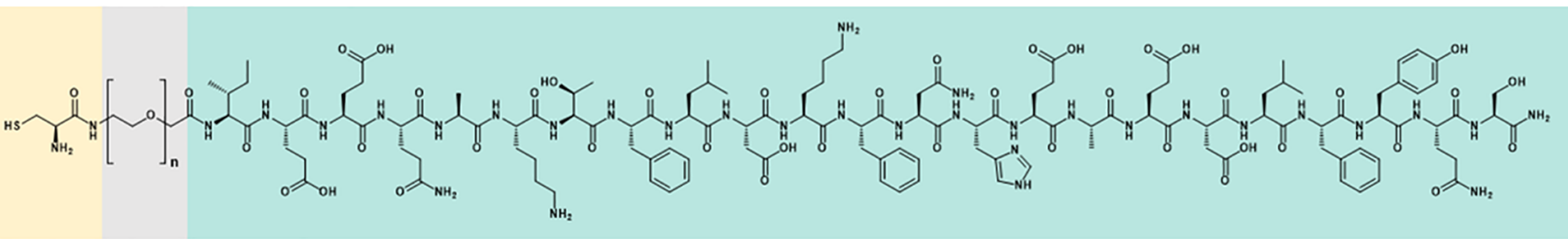

C.

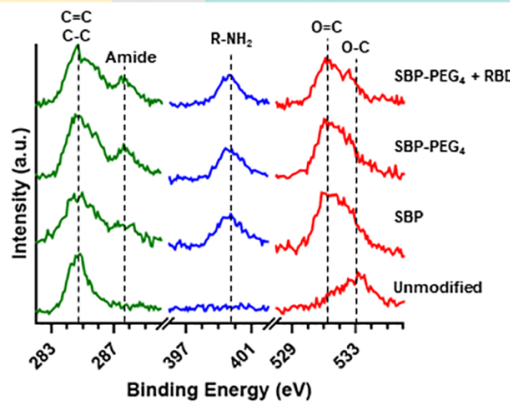

D.

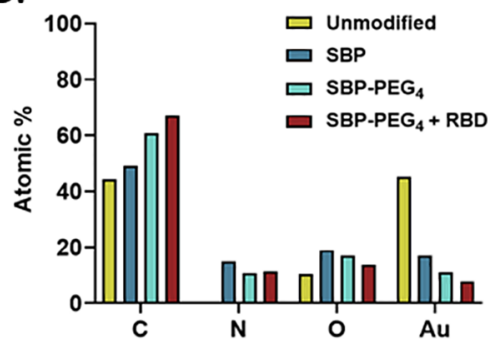

E. (i)

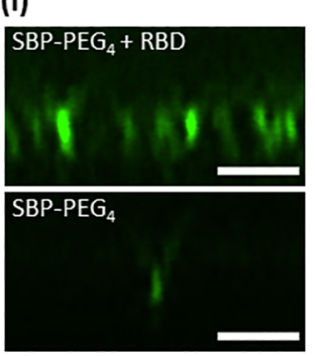

(ii)

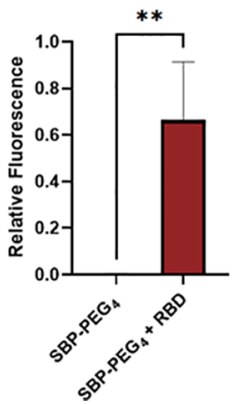

Figure 1. Characterization of peptide-modified sensor for detection of SARS-CoV-2. (A) Schematic of peptide-modified SERS substrates (i) before and (ii) after binding the spike protein of SARS-CoV-2. (B) Sequence and chemical structure of ACE2-derived peptide used to modify surfaces and bind the spike protein. (C) Normalized XPS spectra of SERS substrates showing peptide modification and RBD binding. (D) Atomic composition of surfaces used in (c) showing successful modification and RBD binding. (E) (i) CLSM 3D reconstructed side-view images of immunolabeled SERS surfaces modified with SBP-PEG 4 before and after RBD binding (scale bar $=2 \mu \mathrm{m}$ ). (ii) Relative fluorescence from quantification of integrated density before and after RBD binding $\left(n=4\right.$, area per $\left.n=156 \mu \mathrm{m}^{2}\right)$.

Corresponding binding affinities of peptides were analyzed with steady-state analysis using the HT analysis software. The $K_{\mathrm{d}}$ for SBP

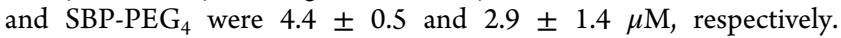
Specificity measurements were carried out using the method described above with the SPB-PEG 4 peptide. Each RBD (SARSCoV-2, SARS-CoV-1, and MERS) was tested at $2 \mu \mathrm{M}$.

Circular Dichroism (CD). CD spectra were obtained on a Chirascan Plus Spectropolarimeter using a $1 \mathrm{~mm}$ path length cuvette. Cysteine-modified peptides were solubilized in PBS at $100 \mu \mathrm{M}$. Spectra were recorded from 200 to $300 \mathrm{~nm}$.

Surface-Enhanced Raman Spectroscopy (SERS). SERS spectra were obtained using a Renishaw inVia Qontor confocal Raman microscope equipped with a CCD camera. A continuous wave laser at $785 \mathrm{~nm}$, a 1200 grooves/mm grating, a $50 \times$ objective with $\mathrm{NA}=0.50$, and $1 \mathrm{~s}$ acquisition times were used for all measurements.

SERS substrates are advantageous for quantification because they can be designed to have homogeneous properties, yielding more reproducible signal enhancement than nanoparticles. ${ }^{29}$ For SERS measurements, commercial gold Silmeco substrates were first cleaned by gently heating to $175^{\circ} \mathrm{C}$ on a hot plate for $10 \mathrm{~min}$. The cleanliness of the substrates was then assessed before use by screening their initial SERS signal for unexpected peaks from contaminants. If necessary, they were cleaned through immersion in a series of solvents, including $1 \mathrm{mM} \mathrm{NaBH}_{4}$ for $3 \mathrm{~min}$ and DMSO or ethanol for $10 \mathrm{~min}$. After cleaning, substrates were either used without modification or functionalized with the cysteine-modified peptides described above. For peptide-functionalization, a $1 \mathrm{mM}$ solution of peptide was prepared (20\% DMSO and $2 \mathrm{mM}$ TCEP in ultrapure water) and allowed to reduce for $30 \mathrm{~min}$. Peptide solution was added to substrates and left overnight for functionalization. Substrates were rinsed with water, backfilled with $100 \mu \mathrm{M} 2$-mercaptoethanol for 30 $\mathrm{min}$, and rinsed with water again before use.
SARS-CoV-2 spike protein, SARS-CoV-2 RBD, SARS-CoV-1 RBD, and MERS-CoV RBD lyophilized powders were reconstituted with water to $0.25 \mathrm{mg} / \mathrm{mL}$ in a buffer, and all solutions were kept frozen or on ice once thawed prior to use. Solutions of $0.1 \%$ BSA were prepared in $1 \times$ PBS. Spike/BSA mixtures were prepared by combining $0.1 \%$ BSA in $1 \times$ PBS with stock spike protein in equal parts. For all SERS experiments, $10 \mu \mathrm{L}$ of protein solution was dropped onto the surface. For experiments on unmodified gold substrates, protein solution was added to the surfaces, and spectra were collected immediately. A power of $2 \mathrm{~mW}$ was used to acquire $20 \mu \mathrm{m} \times 20 \mu \mathrm{m}$ spectral maps with a $1 \mu \mathrm{m}$ step size in the $x y$-plane. Considering the significant heating of the local environment during SERS measurements, photothermal damage to the surface and the sample can be avoided by utilizing near-infrared wavelengths and low powers along with imaging the surface while wet. ${ }^{30,31}$ Laser powers were optimized to avoid potential photodamage to the surfaces. ${ }^{31}$ All SERS measurements were taken on wet substrates. For peptide-functionalized substrates, protein solution was added and allowed to bind for $30 \mathrm{~min}$ before rinsing with water to remove any unbound protein, and SERS measurements were obtained at a power of $570 \mu \mathrm{W}$ with a $10 \mu \mathrm{m} \times$ $10 \mu \mathrm{m}$ map size.

SERS Data Processing and Analysis. Cosmic rays were removed from SERS maps in Windows-based Raman Environment (WiRE) software (version 5.2.10411) from Renishaw. Spectral analysis was performed in MATLAB (R2019b, The Mathworks Inc.). MCR was performed using the PLS Toolbox version 8.7.1 (Eigenvector Research Inc.).

\section{RESULTS AND DISCUSSION}

Surface Modification and Characterization. To design a SERS sensor to detect SARS-CoV-2, we modified SERS 
substrates with a peptide motif derived from the cell surface receptor, ACE2, which binds the RBD of the SARS-CoV-2 spike protein. ${ }^{25,28}$ Displaying the spike-binding peptide (SBP) on SERS substrates will enable the selective capture of SARSCoV-2 from complex media and its detection using SERS (Figure 1A). By synthesizing the SBP with a cysteine residue at its N-terminus (Figure S1), a strong gold-thiol bond attaches this sequence to the gold SERS substrates.

It is ideal to achieve monolayer coverage of the capture molecule in a consistent orientation on the surface. With cysteine-terminated probes, reduction of disulfide bonds before surface modification ensures that the sulfur groups are free to bind to the substrate. Additionally, backfilling with a shortchain thiol can fill the empty spots between probe molecules, encouraging the probe to orient uniformly and in an upright position. ${ }^{32}$ This process can also displace any nonspecifically adsorbed molecules, including contaminants that might remain after cleaning.

Binding of biomolecules to a surface is highly influenced by the chemical properties of the surface, ${ }^{33}$ suggesting that the distance between the surface and the capture peptide recognition sequence could affect its binding affinity to the target molecule. We therefore also examined a second peptide, SBP-PEG 4 (Figure S2), with a $\mathrm{PEG}_{4}$ spacer between the $\mathrm{N}$ terminal cysteine residue and the spike-binding motif (Figure 1B). XPS measurements show successful modification of the substrates with the peptides and RBD binding to the SBP$\mathrm{PEG}_{4}$ surface. From the XPS spectra (Figure 1C) of unmodified versus modified substrates, peaks appear at 288, 399 , and $531 \mathrm{eV}$, indicating amide bonds, primary amines, and $\mathrm{C}=\mathrm{O}$ bonds, respectively. Interestingly, the more predominant amide peak and increased nitrogen-to-gold ratio for the SBP-PEG 4 -modified substrate compared to the SBP-modified substrate indicate that the linker may facilitate better attachment to the surface $(\mathrm{N}: \mathrm{Au}$ ratios are 0.85 and 0.99 for SBP and SBP-PEG 4 , respectively). Upon RBD binding to the SBP-PEG 4 substrate, the XPS spectra maintain a strong amide peak while the $\mathrm{N}: \mathrm{Au}$ ratio increases from 0.99 to 1.45 , indicating that protein is binding to the SBP-PEG -modified $^{-}$ substrate. RBD binding to the SBP-modified substrate was also measured using XPS (Figure S3) with the $\mathrm{N}: \mathrm{Au}$ ratio increasing from 0.85 to 1.31 . Overall, quantification of the atomic composition from the XPS (Figure 1D) supports successful modification and RBD binding as indicated by the increasing $\mathrm{N}: \mathrm{Au}$ ratio after both steps.

To further confirm RBD binding, SBP-PEG -modified $^{-}$ substrates with and without RBD were immunolabeled with anti-RBD fluorescent antibodies and imaged using CLSM to create a $3 \mathrm{D}$ reconstruction of the surface (Figure S4). Sideview images of the substrates (Figure $1 \mathrm{E}(\mathrm{i})$ ) show minimal background signals for SBP-PEG 4 substrates, but after incubation with $\mathrm{RBD}$, increased emission is observed, indicating successful RBD binding. Quantification of the relative fluorescence shows a significant increase after RBD binding (Figure 1E(ii)). SEM images of the substrates (Figure S5) show clustering of pillars after peptide modification, consistent with the known behavior of the SERS substrate. ${ }^{34}$ RBD binding is visualized by immunolabeling with goldnanoparticle-conjugated antibodies (Figure S6).

Enhancing Binding Affinity of the Capture Peptide. We reasoned that introducing a spacer to the peptide between the surface-binding functional group and the analyte binding domain will enable better folding of the peptide-RBD complex and will therefore improve its binding affinity for the RBD. Indeed, BLI measurements of biotinylated versions of SBP (Figure S7) and SBP-PEG 4 (Figure S8) attached to streptavidin-coated BLI tips show a higher response to an increasing spike-RBD concentration from SBP-PEG 4 compared to the SBP sequence (Figure S9). Steady-state analysis of the BLI response indicates that the $\mathrm{PEG}_{4}$ spacer enhances the $K_{\mathrm{d}}$ of the peptide from 4.4 to $2.9 \mu \mathrm{M}$ (Figure $2 \mathrm{~A}$ ), indicating the importance of a spacer between the surface and the spikebinding motif to allow for effective binding of the viral spike protein.
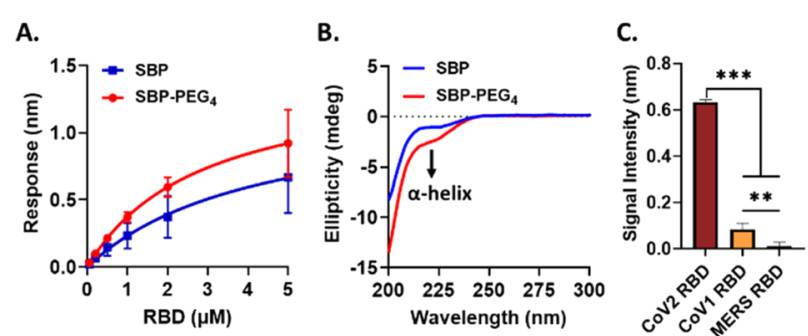

Figure 2. Linker affects binding affinity of ACE2-derived peptides for RBD. (A) Steady-state analysis of BLI data to determine $K_{d}$ values. (B) CD spectra of cysteine-modified peptides with and without a linker comparing the ability of each to form $\alpha$-helical structures. (C) BLI response of SBP-PEG 4 showing specific binding to RBD from SARS-CoV-2 compared to SARS-CoV-1 and MERS.

As the native spike-binding motif adopts an $\alpha$-helix conformation when in the ACE2 protein, we speculate that the spacer might affect the secondary structure of the peptide. $^{28,35} \mathrm{CD}$ measurements show that both SBP and SBP-PEG 4 have a sharp negative peak at $200 \mathrm{~nm}$, indicating significant random coil formation (Figure 2B). Interestingly, SBP-PEG 4 shows a stronger shoulder around $222 \mathrm{~nm}$, which indicates that the linker could promote $\alpha$-helix formation. This difference in helicity correlates with a significantly altered binding affinity, suggesting that the secondary structure plays a critical role in binding the spike protein of SARS-CoV-2 and that enhancing the helicity of the peptide could provide even more improved binding affinity. Of note, the SBP-PEG peptide shows low affinity for other viruses. BLI was used to compare binding between the RBDs of SARS-CoV-2, SARSCoV-1, and MERS (Figure 2C and Figure S10). The peptide shows selectivity for SARS-CoV-2 with significantly higher response compared to SARS-CoV-1, which also binds to the ACE2 receptor. Challenging the peptide with MERS, which does not have an ACE2 binding motif, shows negligible binding. This validates the use of mimetic peptides as capture agents for sensors.

Optimizing SERS Response of Substrates to SARSCoV-2 Spike Protein. SERS performance is dependent on successful peptide modification of the substrate, which requires a clean gold surface. Heating the substrates to $170{ }^{\circ} \mathrm{C}$ helps remove unwanted contaminants before peptide modification, resulting in a reduced SERS background (Figure S11). XPS (Figure S11) and SEM images (Figure S5) show that heating does not damage the substrates. Optimal surface functionalization conditions include the reduction of disulfide bonds between peptide molecules using TCEP and backfilling the surface with 2-mercaptoethanol to orient the peptide more uniformly $^{32}$ on the surface and yield cleaner SERS spectra (Figure S12). The SERS measurements of the SBP and SBP- 
$\mathrm{PEG}_{4}$ peptide-modified gold SERS substrates confirm successful attachment of the peptides to the surfaces (Figure 3A). The SERS spectra of peptide-modified surfaces exhibit
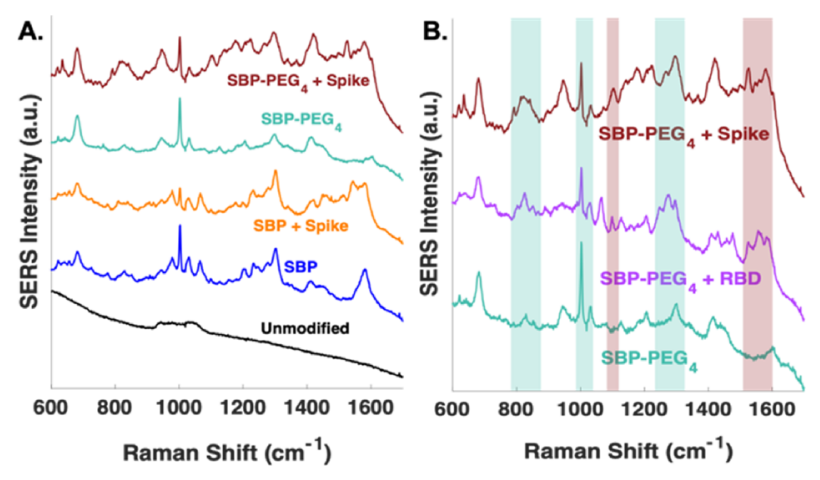

Figure 3. SERS detection of SARS-CoV-2 spike protein and RBD on peptide-modified substrates. (A) SERS spectra of the unmodified substrate and of both peptide-modified substrates before and after addition of $2 \mu \mathrm{M}$ spike protein. (B) Comparison of SERS signal from

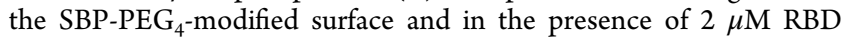
and $2 \mu \mathrm{M}$ full spike, with highlighted regions indicating important spectral similarities associated with the spike/RBD (maroon shading) and SBP-PEG 4 (teal shading). The spectra are offset for clarity.

SERS peaks corresponding to the amino acids in the peptides (Figure 3A). SBP-PEG 4 shows bands at 635, 1002, 1032, 1275, and $1420 \mathrm{~cm}^{-1}$, arising from $\mathrm{C}-\mathrm{S}$ stretching, symmetric ring breathing of phenylalanine, in-plane bending of phenylalanine, phenylalanine/tyrosine $\mathrm{CH}_{2}$ wagging, and $\mathrm{CH}_{2}$ deformation of cysteine, respectively. ${ }^{36-38}$ Figure $3 \mathrm{~A}$ further shows that the SARS-CoV-2 spike protein elicits significant changes in the observed SERS spectrum. When treated with the SARS-CoV-2 spike protein, the SBP-PEG 4 surface shows more significant and additional spectral changes compared to the peptide without the spacer (Figure 3A), in agreement with its higher binding affinity for the RBD (Figure 2).

Addition of the spike protein to the SBP-PEG 4 surface shows peaks assigned to the protein: $\mathrm{C}-\mathrm{H}$ stretching or $\mathrm{N}-\mathrm{H}$ deformation at $793 \mathrm{~cm}^{-1}$, phenylalanine out-of-plane bending at $842 \mathrm{~cm}^{-1}, \alpha$-helical skeletal vibration at $946 \mathrm{~cm}^{-1}, \mathrm{C}-\mathrm{N}$ stretching at $1103 \mathrm{~cm}^{-1}$, tyrosine vibration at $1175 \mathrm{~cm}^{-1}, \mathrm{C}-\mathrm{C}$ stretching of tyrosine and phenylalanine at $1223 \mathrm{~cm}^{-1}$, tryptophan rocking at $1267 \mathrm{~cm}^{-1}$, amide III $\alpha$-helix vibration at $1293 \mathrm{~cm}^{-1}$, tryptophan $\mathrm{C}_{\alpha}-\mathrm{H}$ deformation at $1339 \mathrm{~cm}^{-1}$, $\mathrm{CH}_{3}$ symmetric stretching at $1369 \mathrm{~cm}^{-1}, \mathrm{NH}_{3}{ }^{+}$deformation of lysine at $1526 \mathrm{~cm}^{-1}$, indole ring of tryptophan at $1559 \mathrm{~cm}^{-1}$, tryptophan aromatic ring stretching at $1580 \mathrm{~cm}^{-1}$, and phenylalanine or tyrosine $\mathrm{C}-\mathrm{C}$ ring stretching at 1601 $\mathrm{cm}^{-1}$. 36,37,39-41 These assignments are consistent with the structure of the spike protein and its RBD. ${ }^{35}$

The observed SERS features in conjunction with the BLI data indicate that SBP-PEG 4 is the preferred capture molecule. The SERS signatures of SARS-CoV-2 RBD versus the full spike protein on an SBP-PEG 4 surface show a high degree of similarity (Figure $3 \mathrm{~B}$ ), suggesting that the majority of the signal from the full spike originates from the RBD. ${ }^{42-44}$ As noted in Figure 3B, addition of either the spike or the RBD triggers the appearance of similar peaks (highlighted in maroon), such as the bands around $1600 \mathrm{~cm}^{-1}$ from tryptophan, along with changes in the relative intensities of bands from the peptide itself (highlighted in teal), supporting a change in orientation associated with protein binding.
The SBP-PEG 4 surface demonstrates specificity for SARSCoV-2 RBD, as opposed to the RBD of two other human coronaviruses, SARS-CoV-1 and MERS-CoV, in agreement with the previously discussed BLI results. In Figure 4, the sensor yields an intense SERS signal from $5 \mu \mathrm{M}$ SARS-CoV-2 RBD but no response from of SARS-CoV-1 RBD and MERS$\mathrm{CoV}$ RBD under the same conditions.

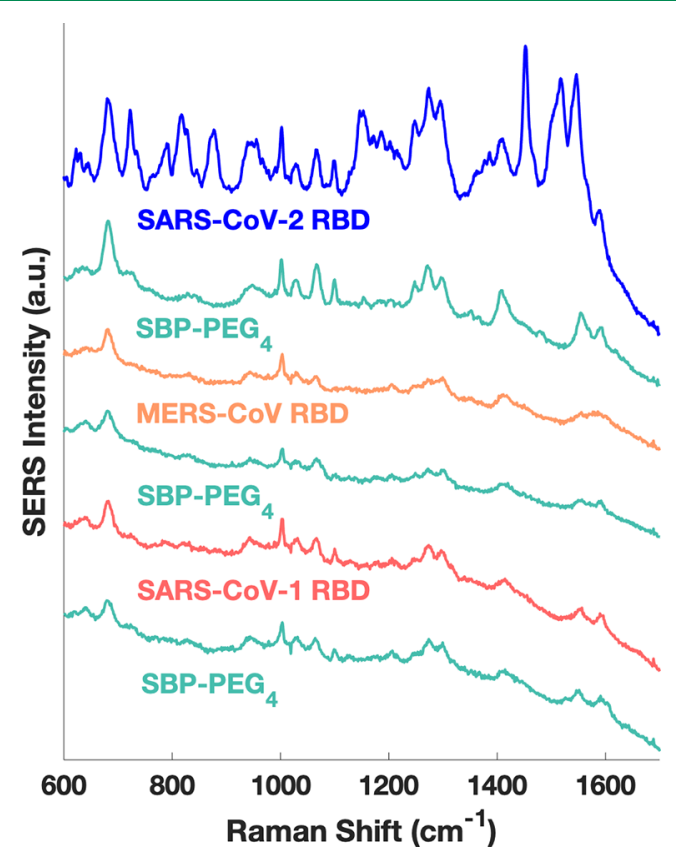

Figure 4. Specificity of the SBP-PEG 4 SERS sensor for SARS-CoV-2 RBD (blue) versus SARS-CoV-1 RBD (red) and MERS-CoV RBD (orange). The peptide surfaces (teal) prior to treatment with each $\mathrm{RBD}(5 \mu \mathrm{M})$ are shown below each spectrum, respectively. The spectra are offset for clarity.

Evaluating Selectivity and Quantitative Capabilities of the SERS Sensor. Figure 5 shows the SERS spectra obtained from SBP-PEG 4 -functionalized substrates treated with either $2 \mu \mathrm{M}$ spike protein, $15 \mu \mathrm{M}$ bovine serum albumin (BSA), or a spike and BSA mixture. BSA was mixed with the SARS-CoV-2 spike protein in an 8:1 ratio to mimic the protein-rich environment of saliva and blood. In all of these experiments, the SERS signal was acquired from each SBP$\mathrm{PEG}_{4}$-modified surface before and after treatment with protein. In Figure 5A, the SERS spectrum in the presence of spike protein shows distinct differences from the peptide-modified surface, notably the feature around $1600 \mathrm{~cm}^{-1}$ (as indicated by the maroon box in Figure 5A), which is likely associated with tryptophan residues present in the $\mathrm{RBD}$ of the spike protein but not present in SBP-PEG 4 . These differences are unique to the surfaces containing spike proteins and are not observed in the presence of BSA alone. This competition assay assessed the selectivity of the SBP-PEG 4 -modified surface, demonstrating the advantage of the capture peptide.

MCR was used to generate a model capturing the spectral changes observed from the spike protein binding to the SBP$\mathrm{PEG}_{4}$-modified SERS substrate. The stochastic nature of molecules interacting with hotspots on the surface is known to produce some variance in the SERS spectra. MCR generates components representative of the average spectra observed in all the data. The calibration data consisted of SERS maps from an SBP-PEG 4 -modified SERS substrate challenged with a high 

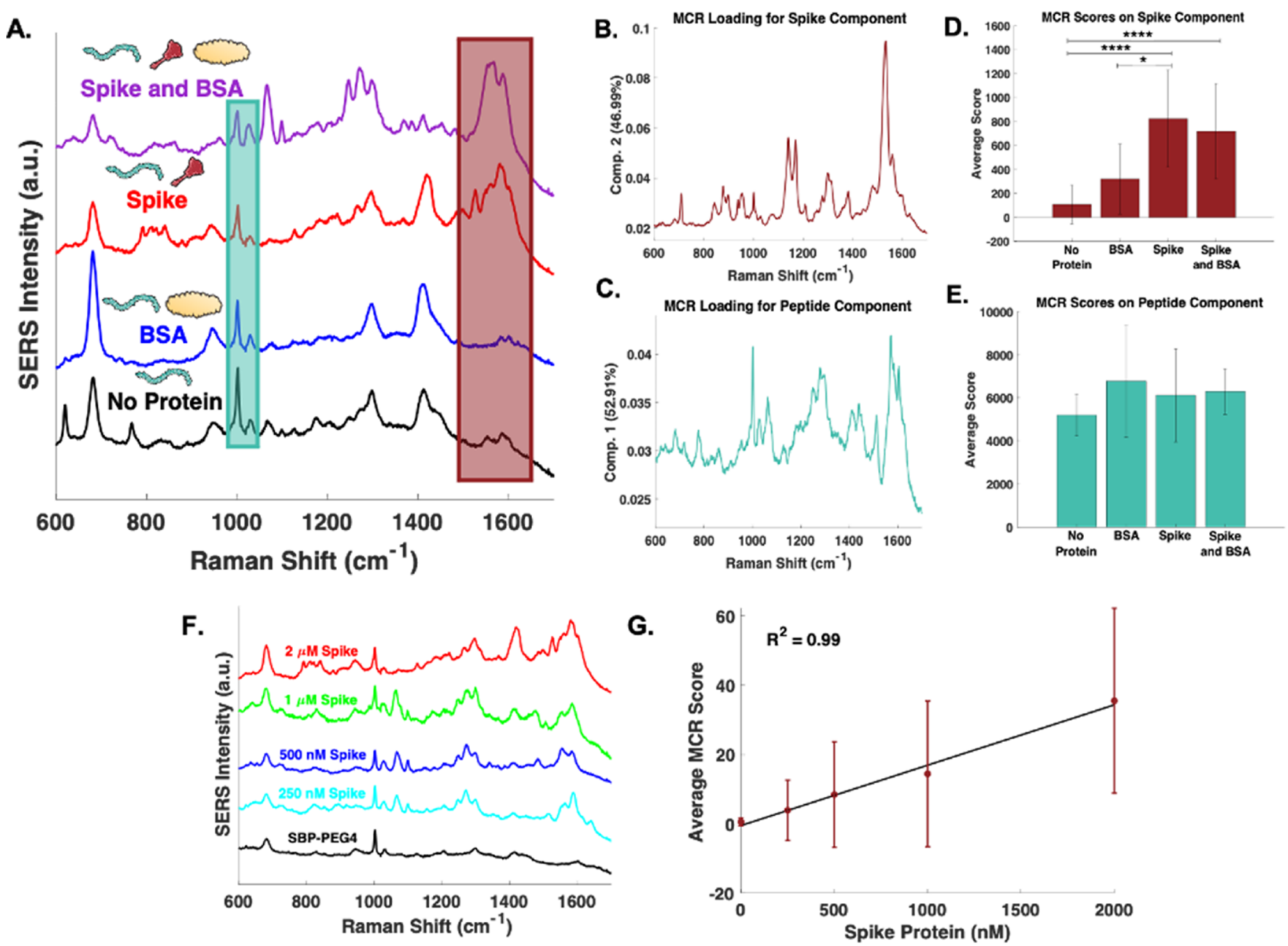

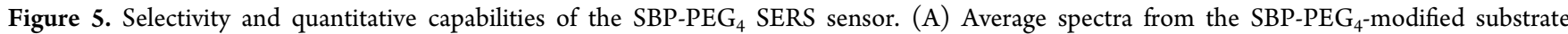
treated with no protein, $15 \mu \mathrm{M}$ BSA, $2 \mu \mathrm{M}$ spike, and $8 \mu \mathrm{M}$ BSA plus $1 \mu \mathrm{M}$ spike ( $8: 1$ mixture). (B) MCR component 2 , representing the SERS signature of the spike protein. (C) MCR component 1, representing the SERS signature of the peptide. (D) MCR scores on component 2 for the

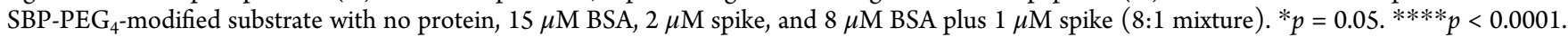
(E) MCR scores on component 1 for the SPB-PEG4-modified substrate with no protein, $8 \mu \mathrm{M}$ BSA, $2 \mu \mathrm{M}$ spike, and $8 \mu \mathrm{M}$ BSA plus $1 \mu \mathrm{M}$ spike (8:1 mixture). (F) Average spectra (normalized to $1002 \mathrm{~cm}^{-1}$ ) from the $S B P-\mathrm{PEG}_{4}$-modified substrate treated with varying concentrations of spike protein. (G) SERS-based calibration curve for the spike using MCR scores on the spike component, showing a limit of detection of $300 \mathrm{nM}$. The spectra are offset for clarity.

concentration of the spike protein, generating a twocomponent model. The loadings derived from the MCR model are consistent with the spectral changes observed from the spike protein (Figure 5B) and the SBP-PEG 4 modified SERS substrate (Figure 5C). The strong spectral feature between 1500 and $1600 \mathrm{~cm}^{-1}$ in the loading for the second component (Figure 5B) correlates to a similar feature present in the average spectra of the spike-containing samples (Figure $5 \mathrm{~A})$. The emergence of signal in this region appears to be the key indicator of spike binding. Data collected on four different days across four different substrates, which produced a total of five spike maps, five BSA maps, eight mixture maps, and 18 maps with no protein, were analyzed with the resulting model. During the process, Grubbs' test removed one out of the 18 total peptide maps as an outlier from the validation set. Figure 5D shows that the addition of both the spike and the spike/ BSA mixture causes a statistically significant increase $(p<$ 0.0001 ) in the sample's score on the spike protein component compared to the surface with no protein. Meanwhile, Figure $5 \mathrm{E}$ shows that the scores on the peptide component do not change upon the addition of the protein solutions. The data also exhibits a significant difference $(p=0.05)$ between the BSA-treated and the spike-treated samples on the spike component. The score of BSA on the spike component likely represents the small amount of overlap between an off-target protein SERS signal and the SERS spectra of the spike protein, validating that the peptide provides the sensor with selectivity for the SARS-CoV-2 spike protein. Upon addition of the mixture and subsequent rinsing of the surface, the BSA is likely washed away, while the spike protein remains bound to the peptide on the surface. Additionally, the BSA may promote binding of the spike protein to the peptide, as indicated by the similar spike component scores of the $2 \mu \mathrm{M}$ spike and the mixture containing only $1 \mu \mathrm{M}$ spike. BSA is typically used as a blocking agent to prevent nonspecific adsorption; ${ }^{45}$ however, since the surface is modified with both a peptide and a backfilling agent, this surface effect is unlikely to affect binding. Instead, the BSA may be acting as a molecular chaperone for the spike protein, preventing aggregation and encouraging 
proper folding, ${ }^{46}$ thus ensuring conditions conducive to peptide-spike binding.

It is important to note that this sensor is designed to mimic the ACE2 receptor found in humans and that the model selects for the spike protein of SARS-CoV-2. Because variants of the virus are expected to also infect cells through the ACE2 receptor, this sensor is expected to also bind to the variants. For the sensing of variants, the model may need to be retrained, particularly in the case where the $R B D$ in the variant is altered. ${ }^{4-49}$

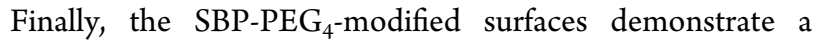
linear SERS response as a function of SARS-CoV-2 spike protein concentration. SERS maps were acquired from SBP$\mathrm{PEG}_{4}$ substrates treated with five different concentrations of spike protein. We observed that the heterogeneity of the SERS substrate required analysis of an average response from a 10 $\mu \mathrm{m} \times 10 \mu \mathrm{m}$ area for reliable detection. This could also be achieved with a larger illumination area in a practical device. The spectra from five maps from each surface were averaged and normalized to the height of the phenylalanine peak at 1002 $\mathrm{cm}^{-1}$ as an internal standard (Figure $5 \mathrm{~F}$ ). The average scores for the normalized spectra on the previously discussed MCR model (Figure 5B,D) generated a calibration curve (Figure $5 G$ ) for the SARS-CoV-2 spike protein on the SBP-PEG ${ }_{4}^{-}$ modified surface, giving a limit of detection (LOD) of $300 \mathrm{nM}$. Here, LOD is calculated as $3 S_{y} / m$, where $S_{y}$ is the standard deviation in $y$ and $m$ is the slope of the calibration curve. The SBP-PEG 4 sensor has an LOD about two times lower than that of an unmodified surface (Figure S13) and demonstrates better selectivity, as shown by the competition assay (Figure 5).

\section{CONCLUSIONS}

The use of a SERS substrate modified with a virus-capture peptide provides selective detection of SARS-CoV-2 proteins in complex media. From starting with a clean surface to implementing an effective target-binding sequence, designing a functional peptide-based SERS sensor requires careful attention to detail and consideration of several factors. Notably, using a linker to offset the protein-binding sequence of the peptide from the surface impacts binding, potentially by altering the conformation of the peptide. The SBP-PEG 4 SERS sensor shows improved detection of the SARS-CoV-2 spike protein at lower concentrations compared to the unmodified surface. Consistent with previous findings, the SERS signal of this protein appears to be derived from the RBD. Variants of the SARS-CoV-2 virus are reported to have mutations in the $\mathrm{RBD},{ }^{47-49}$ but since SBP-PEG 4 mimics the natural binding site of the virus, this SERS sensor will likely still bind these variants, and the model could be retrained to detect them without engineering a new sensor. Overall, the improved LOD and the selectivity of the sensor provide the basis for the utilization of a peptide-based SERS assay for detecting SARS-CoV-2 as well as other emerging viruses in the future.

\section{ASSOCIATED CONTENT}

\section{SI Supporting Information}

The Supporting Information is available free of charge at https://pubs.acs.org/doi/10.1021/acssensors.1c01344.

Peptide names and sequences, Cys-SBP peptide, Cys$\mathrm{PEG}_{4}$-SBP peptide, XPS of RBD binding SBP substrate, confocal images of immunolabeled substrates, SEM of SERS substrates before and after modification, SEM of immunolabeled substrate, biotin-SBP peptide, biotin$\mathrm{PEG}_{4}$-SBP peptide, BLI kinetics, peptide specificity BLI curves, XPS and SERS characterization of heat cleaning substrates, SERS characterization of backfilling, and SERS response of spike protein on unmodified substrates (PDF)

\section{AUTHOR INFORMATION}

\section{Corresponding Authors}

Ronit Freeman - Department of Applied Physical Sciences, University of North Carolina, Chapel Hill, North Carolina 27599, United States; 이이.org/0000-0001-5960-6689; Email: ronifree@email.unc.edu

Zachary D. Schultz - Department of Chemistry and Biochemistry, The Ohio State University, Columbus, Ohio 43210, United States; 이이.orid.org/0000-0003-1741-8801; Email:schultz.133@osu.edu

\section{Authors}

Taylor D. Payne - Department of Chemistry and Biochemistry, The Ohio State University, Columbus, Ohio 43210, United States

Stephen J. Klawa - Department of Applied Physical Sciences, University of North Carolina, Chapel Hill, North Carolina 27599, United States; (1) orcid.org/0000-0003-2416-7721

Tengyue Jian - Department of Applied Physical Sciences, University of North Carolina, Chapel Hill, North Carolina 27599, United States; (1) orcid.org/0000-0002-4463-5492

Sang Hoon Kim - Department of Applied Physical Sciences, University of North Carolina, Chapel Hill, North Carolina 27599, United States

Micah J. Papanikolas - Department of Applied Physical Sciences, University of North Carolina, Chapel Hill, North Carolina 27599, United States; (1) orcid.org/0000-00033149-312X

Complete contact information is available at:

https://pubs.acs.org/10.1021/acssensors.1c01344

\section{Author Contributions}

${ }^{\S}$ T.D.P. and S.J.K. contributed equally.

\section{Notes}

The authors declare the following competing financial interest(s): We have a patent pending related to this manuscript.

\section{ACKNOWLEDGMENTS}

This work was supported by The Ohio State University and the University of North Carolina (UNC) at Chapel Hill. The authors acknowledge financial support from the Research Corporation for Science Advancement. R.F. also acknowledges support from the North Carolina Policy Collaboratory at the University of North Carolina at Chapel Hill with funding from the North Carolina Coronavirus Relief Fund established and appropriated by the North Carolina General Assembly. Z.S. acknowledges support by the Ohio State University Comprehensive Cancer Center and the National Institutes of Health under grant number P30 CA016058. Mass spectrometry was performed at the UNC Mass Spectrometry Core Laboratory at CRITCL (Chemical Research Instrumentation Teaching and Core Labs) in the Department of Chemistry. XPS and SEM were performed at the Chapel Hill Analytical and Nanofabrication Laboratory (CHANL), a member of the 
North Carolina Research Triangle Nanotechnology Network (RTNN) funded by the National Science Foundation (NSF) (grant ECCS-2025064) as part of the National Nanotechnology Coordinated Infrastructure (NNCI). CLSM images were taken at UNC Microscopy Services Laboratory (MSL). The Microscopy Services Laboratory, Department of Pathology and Laboratory Medicine, and CD and BLI measurements taken at the UNC Macromolecular Interactions Facility are supported in part by P30 CA016086 Cancer Center Core Support Grant to the UNC Lineberger Comprehensive Cancer Center funded by the National Cancer Institute. The content is solely the responsibility of the authors and does not necessarily represent the official views of the National Institutes of Health. We thank Peter Harris for the graphic illustrations incorporated into the manuscript.

\section{REFERENCES}

(1) World Health Organization Coronavirus Disease (COVID-19) Dashboard; World Health Organization: 2021 https://covid19.who. int.

(2) Winter, L. False Negatives in Quick COVID-19 Test Near 15 Percent: Study; LabX Media Group: 2020.

(3) Smith, E.; Dent, G. Modern Raman Spectroscopy- A Practical Approach; John Wiley \& Sons, Ltd.: England, 2005.

(4) Jeanmaire, D. L.; Van Duyne, R. P. Surface Raman Spectroelectrochemistry. J. Electroanal. Chem. Interfacial Electrochem. 1977, 84, 1-20.

(5) Langer, J.; Jimenez de Aberasturi, D.; Aizpurua, J.; AlvarezPuebla, R. A.; Auguié, B.; Baumberg, J. J.; Bazan, G. C.; Bell, S. E. J.; Boisen, A.; Brolo, A. G.; Choo, J.; Cialla-May, D.; Deckert, V.; Fabris, L.; Faulds, K.; García de Abajo, F. J.; Goodacre, R.; Graham, D.; Haes, A. J.; Haynes, C. L.; Huck, C.; Itoh, T.; Käll, M.; Kneipp, J.; Kotov, N. A.; Kuang, H.; Le Ru, E. C.; Lee, H. K.; Li, J.-F.; Ling, X. Y.; Maier, S. A.; Mayerhöfer, T.; Moskovits, M.; Murakoshi, K.; Nam, J.-M.; Nie, S.; Ozaki, Y.; Pastoriza-Santos, I.; Perez-Juste, J.; Popp, J.; Pucci, A.; Reich, S.; Ren, B.; Schatz, G. C.; Shegai, T.; Schlücker, S.; Tay, L.-L.; Thomas, K. G.; Tian, Z.-Q.; Van Duyne, R. P.; Vo-Dinh, T.; Wang, Y.; Willets, K. A.; Xu, C.; Xu, H.; Xu, Y.; Yamamoto, Y. S.; Zhao, B.; Liz-Marzán, L. M. Present and Future of Surface-Enhanced Raman Scattering. ACS Nano 2020, 14, 28-117.

(6) Nguyen, A. H.; Peters, E. A.; Schultz, Z. D. Bioanalytical Applications of Surface-Enhanced Raman Spectroscopy: De Novo Molecular Identification. Rev. Anal. Chem. 2017, 36, 20160037.

(7) Tate, J.; Ward, G. Interferences in Immunoassay. Clin. Biochem. Rev. 2004, 25, 105-120.

(8) Wang, F.; Cao, S.; Yan, R.; Wang, Z.; Wang, D.; Yang, H. Selectivity/Specificity Improvement Strategies in Surface-Enhanced Raman Spectroscopy Analysis. Sensors 2017, 17, 2689.

(9) Driskell, J. D.; Uhlenkamp, J. M.; Lipert, R. J.; Porter, M. D. Surface-Enhanced Raman Scattering Immunoassays Using a Rotated Capture Substrate. Anal. Chem. 2007, 79, 4141-4148.

(10) Karn-orachai, K.; Sakamoto, K.; Laocharoensuk, R.; Bamrungsap, S.; Songsivilai, S.; Dharakul, T.; Miki, K. Extrinsic Surface-Enhanced Raman Scattering Detection of Influenza A Virus Enhanced by Two-Dimensional Gold@silver Core-Shell Nanoparticle Arrays. RSC Adv. 2016, 6, 97791-97799.

(11) Chen, H.; Park, S.-G.; Choi, N.; Kwon, H.-J.; Kang, T.; Lee, M.K.; Choo, J. Sensitive Detection of SARS-CoV-2 Using a SERS-Based Aptasensor. ACS Sens. 2021, 2378.

(12) Negri, P.; Chen, G.; Kage, A.; Nitsche, A.; Naumann, D.; Xu, B.; Dluhy, R. A. Direct Optical Detection of Viral Nucleoprotein Binding to an Anti-Influenza Aptamer. Anal. Chem. 2012, 84, 55015508 .

(13) Fu, X.; Cheng, Z.; Yu, J.; Choo, P.; Chen, L.; Choo, J. A SERSBased Lateral Flow Assay Biosensor for Highly Sensitive Detection of HIV-1 DNA. Biosens. Bioelectron. 2016, 78, 530-537.
(14) Ma, H.; Tang, X.; Liu, Y.; Han, X. X.; He, C.; Lu, H.; Zhao, B. Surface-Enhanced Raman Scattering for Direct Protein Function Investigation: Controlled Immobilization and Orientation. Anal. Chem. 2019, 91, 8767-8771.

(15) Lussier, F.; Thibault, V.; Charron, B.; Wallace, G. Q.; Masson, J.-F. Deep Learning and Artificial Intelligence Methods for Raman and Surface-Enhanced Raman Scattering. TrAC, Trends Anal. Chem. 2020, 124, 115796

(16) Lussier, F.; Missirlis, D.; Spatz, J. P.; Masson, J.-F. MachineLearning-Driven Surface-Enhanced Raman Scattering Optophysiology Reveals Multiplexed Metabolite Gradients Near Cells. ACS Nano 2019, DOI: 10.1021 /acsnano.8b07024.

(17) Thrift, W.; Bhattacharjee, A.; Hochbaum, A. I.; Ragan, R.; Nguyen, C.; Whiteson, K. Robust SERS Spectral Analysis for Quantitative Detection of Pyocyanin in Biological Fluids. In Biosensing and Nanomedicine X; Mohseni, H., Agahi, M. H., Razeghi, M. Eds.; SPIE: San Diego, United States, 2017; p 4, DOI: 10.1117/ 12.2267958

(18) Thrift, W. J.; Ragan, R. Quantification of Analyte Concentration in the Single Molecule Regime Using Convolutional Neural Networks. Anal. Chem. 2019, 91, 13337-13342.

(19) Goodacre, R.; Graham, D.; Faulds, K. Recent Developments in Quantitative SERS: Moving towards Absolute Quantification. $\operatorname{Tr} A C$, Trends Anal. Chem. 2018, 102, 359-368.

(20) Pérez-Jiménez, A. I.; Lyu, D.; Lu, Z.; Liu, G.; Ren, B. SurfaceEnhanced Raman Spectroscopy: Benefits, Trade-Offs and Future Developments. Chem. Sci. 2020, 11, 4563-4577.

(21) Nguyen, A.; Schultz, Z. D. Quantitative Online Sheath-Flow Surface Enhanced Raman Spectroscopy Detection for Liquid Chromatography. Analyst 2016, 141, 3630-3635.

(22) Bell, S. E. J.; Mackle, J. N.; Sirimuthu, N. M. S. Quantitative Surface-Enhanced Raman Spectroscopy of Dipicolinic Acid-towards Rapid Anthrax Endospore Detection. Analyst 2005, 130, 545-549.

(23) Villa, J. E. L.; Poppi, R. J. A Portable SERS Method for the Determination of Uric Acid Using a Paper-Based Substrate and Multivariate Curve Resolution. Analyst 2016, 141, 1966-1972.

(24) Mamián-López, M. B.; Poppi, R. J. Standard Addition Method Applied to the Urinary Quantification of Nicotine in the Presence of Cotinine and Anabasine Using Surface Enhanced Raman Spectroscopy and Multivariate Curve Resolution. Anal. Chim. Acta 2013, 760, $53-59$.

(25) Wrapp, D.; Wang, N.; Corbett, K. S.; Goldsmith, J. A.; Hsieh, C.-L.; Abiona, O.; Graham, B. S.; McLellan, J. S. Cryo-EM Structure of the 2019-NCoV Spike in the Prefusion Conformation. Science 2020, 367, 1260-1263.

(26) Yuan, M.; Wu, N. C.; Zhu, X.; Lee, C.-C. D.; So, R. T. Y.; Lv, H.; Mok, C. K. P.; Wilson, I. A. A Highly Conserved Cryptic Epitope in the Receptor Binding Domains of SARS-CoV-2 and SARS-CoV. Science 2020, 368, 630-633.

(27) Nguyen, H. L.; Lan, P. D.; Thai, N. Q.; Nissley, D. A.; O’Brien, E. P.; Li, M. S. Does SARS-CoV-2 Bind to Human ACE2 More Strongly Than Does SARS-CoV? J. Phys. Chem. B 2020, 124, 73367347.

(28) Zhang, G.; Pomplun, S.; Loftis, A. R.; Tan, X.; Loas, A.; Pentelute, B. L. Investigation of ACE2 N-Terminal Fragments Binding to SARS-CoV-2 Spike RBD; preprint. BioRxiv Cold Spring Harbor Laboratory 2020, DOI: 10.1101/2020.03.19.999318.

(29) Asiala, S. M.; Schultz, Z. D. Characterization of Hotspots in a Highly Enhancing SERS Substrate. Analyst 2011, 136, 4472.

(30) Takase, M.; Nabika, H.; Hoshina, S.; Nara, M.; Komeda, K.; Shito, R.; Yasuda, S.; Murakoshi, K. Local Thermal Elevation Probing of Metal Nanostructures during Laser Illumination Utilizing SurfaceEnhanced Raman Scattering from a Single-Walled Carbon Nanotube. Phys. Chem. Chem. Phys. 2013, 15, 4270.

(31) Zeng, Z.-C.; Wang, H.; Johns, P.; Hartland, G. V.; Schultz, Z. D. Photothermal Microscopy of Coupled Nanostructures and the Impact of Nanoscale Heating in Surface-Enhanced Raman Spectroscopy. J. Phys. Chem. C 2017, 121, 11623-11631. 
(32) Oberhaus, F. V.; Frense, D.; Beckmann, D. Immobilization Techniques for Aptamers on Gold Electrodes for the Electrochemical Detection of Proteins: A Review. Biosensors 2020, 10, 45.

(33) Gibbs, J.; Vessels, M.; Rothenberg, M. Immobilization Principles - Selecting the Surface for ELISA Assays; Corning Incorporated, Life Sciences: 2017.

(34) Schmidt, M. S.; Hübner, J.; Boisen, A. Large Area Fabrication of Leaning Silicon Nanopillars for Surface Enhanced Raman Spectroscopy. Adv. Mater. 2012, 24, OP11-OP18.

(35) Lan, J.; Ge, J.; Yu, J.; Shan, S.; Zhou, H.; Fan, S.; Zhang, Q.; Shi, X.; Wang, Q.; Zhang, L.; Wang, X. Structure of the SARS-CoV-2 Spike Receptor-Binding Domain Bound to the ACE2 Receptor. Nature 2020, 581, 215-220.

(36) Szekeres, G. P.; Kneipp, J. SERS Probing of Proteins in Gold Nanoparticle Agglomerates. Front. Chem. 2019, 7, 30.

(37) Freire, P. T. C.; Barboza, F. M.; Lima, J. A.; Melo, F. E. A.; Filho, J. M. Raman Spectroscopy of Amino Acid Crystals. In Raman Spectroscopy and Applications; Maaz, K. Ed.; InTech: 2017; DOI: $10.5772 / 65480$.

(38) Negri, P.; Schultz, Z. D. Online SERS Detection of the 20 Proteinogenic L-Amino Acids Separated by Capillary Zone Electrophoresis. Analyst 2014, 139, 5989-5998.

(39) Rygula, A.; Majzner, K.; Marzec, K. M.; Kaczor, A.; Pilarczyk, M.; Baranska, M. Raman Spectroscopy of Proteins: A Review: Raman Spectroscopy of Proteins. J. Raman Spectrosc. 2013, 44, 1061-1076.

(40) Lin, V. J. C.; Koenig, J. L. Raman Studies of Bovine Serum Albumin. Biopolymers 1976, 15, 203-218.

(41) Peticolas, W. L. Raman Spectroscopy of DNA and Proteins. In Methods in Enzymology; Elsevier: 1995; Vol. 246, pp. 389-416, DOI: 10.1016/0076-6879(95)46019-5.

(42) Wang, H.; Carrier, S. L.; Park, S.; Schultz, Z. D. Selective TERS Detection and Imaging through Controlled Plasmonics. Faraday Discuss. 2015, 178, 221-235.

(43) Wang, H.; Schultz, Z. D. TERS Detection of $\alpha_{\mathrm{V}} \beta_{3}$ Integrins in Intact Cell Membranes. ChemPhysChem 2014, 15, 3944-3949.

(44) Xiao, L.; Wang, H.; Schultz, Z. D. Selective Detection of RGDIntegrin Binding in Cancer Cells Using Tip Enhanced Raman Scattering Microscopy. Anal. Chem. 2016, 88, 6547-6553.

(45) Lichtenberg, J. Y.; Ling, Y.; Kim, S. Non-Specific Adsorption Reduction Methods in Biosensing. Sensors 2019, 19, 2488.

(46) Finn, T. E.; Nunez, A. C.; Sunde, M.; Easterbrook-Smith, S. B. Serum Albumin Prevents Protein Aggregation and Amyloid Formation and Retains Chaperone-like Activity in the Presence of Physiological Ligands. J. Biol. Chem. 2012, 287, 21530-21540.

(47) Yuan, M.; Huang, D.; Lee, C.-C. D.; Wu, N. C.; Jackson, A. M.; Zhu, X.; Liu, H.; Peng, L.; van Gils, M. J.; Sanders, R. W.; Burton, D. R.; Reincke, S. M.; Prüss, H.; Kreye, J.; Nemazee, D.; Ward, A. B.; Wilson, I. A. Structural and Functional Ramifications of Antigenic Drift in Recent SARS-CoV-2 Variants. Science 2021, 818.

(48) Zhang, J.; Cai, Y.; Xiao, T.; Lu, J.; Peng, H.; Sterling, S. M.; Walsh, R. M.; Rits-Volloch, S.; Sliz, P.; Chen, B. Structural Impact on SARS-CoV-2 Spike Protein by D614G Substitution. bioRxiv Cold Spring Harbor Laboratory: 2020, 525-530, DOI: 10.1101/ 2020.10.13.337980.

(49) Singh, J.; Samal, J.; Kumar, V.; Sharma, J.; Agrawal, U.; Ehtesham, N. Z.; Sundar, D.; Rahman, S. A.; Hira, S.; Hasnain, S. E. Structure-Function Analyses of New SARS-CoV-2 Variants B.1.1.7, B.1.351 and B.1.1.28.1: Clinical, Diagnostic, Therapeutic and Public Health Implications. Viruses 2021, 13, 439. 